\title{
COMPARATIVE ASSESSMENT OF THERMAL RESISTANCE OF PALM STEARIN AND HIGH OLEIC BLENDED OIL WHEN SUBJECTED TO FRYING PRACTICE IN FAST FOOD RESTAURANTS
}

\section{MINGMING HU*; KAILIN PAN**; YUETING NIU**; SHIQING CHEN"** JUN HAO YOONG‡; AZMIL HAIZAM AHMAD TARMIZI ${ }^{\ddagger}$ and GUOWEN ZHANG*}

\begin{abstract}
In this study, the thermal stability of palm frying oil (palm stearin, POs) and a formulated high oleic frying oil (high oleic blended oil, $\mathrm{HOBO}$ ) in China was evaluated under simulated frying protocol in fast food restaurants. A series of physico-chemical properties of the oils were measured including change of colour, acid value $(A V)$, peroxide value $(P V), \mathrm{p}$-anisidine value ( $\mathrm{p}-A V)$, total oxidation (TOTOX $=2 P V+\mathrm{p}-A V$ ), fatty acids composition (FAC), iodine value (IV), carbonyl group value (CGV), total polar compounds (TPC), oxidative stability index (OSI) and vitamin E content. The frying experiments were conducted at $170 \pm 5^{\circ} \mathrm{C}$ for five consecutive days where French fries were fried in five cycles hourly for $12 \mathrm{hr}$ a day. The results indicated that $P O s$ gave higher physical and chemical stability than $\mathrm{HOBO}$ under extended frying conditions, considering its relatively lower increase in TPC, $\mathrm{p}-A V$, TOTOX and CGV, lesser decrease in C18:2/C16:0 and IV, and longer induction period. Based on the results, it can be conjectured that POs is a more preferred choice for deep fat frying with lower deterioration rate.
\end{abstract}

Keywords: palm stearin, high oleic oil, frying performances, deep fat frying.

Date received: 16 August 2019; Sent for revision: 20 August 2019; Accepted: 16 December 2019.

\section{INTRODUCTION}

Deep fat frying is one of the most well-accepted methods in food preparation, owing to the fact that

* State Key Laboratory of Food Science and Technology, 235 Nanjing E Rd, Qingshanhu, Nanchang University, Nanchang 330047, People's Republic of China.

E-mail: gwzhang@ncu.edu.cn

** Palm Oil Research and Technical Service Institute of Malaysian Palm Oil Board, Level 2, No. 18 Lane 88, Yuanshan road, Xinzhuang Industrial Park, Minhang, Shanghai 201108, People's Republic of China.

‡ Malaysian Palm Oil Board, 6 Persiaran Institusi, Bandar Baru Bangi,

43000 Kajang, Selangor, Malaysia. fried food provides unique aroma, taste, golden colour and crispy texture that cannot be found in other cooking techniques (Aladedunye, 2015). Essentially, deep fat frying involves immersion of food in hot oil at temperatures from $150^{\circ} \mathrm{C}$ to $190^{\circ} \mathrm{C}$ (Aniolowska and Kita, 2016; Yu et al., 2016). During the frying process, oil undergoes a series of reactions which can produce unwanted breakdown compounds (Del Re and Jorge, 2006). These reactions mainly comprised of oxidation, hydrolysis and polymerisation, giving rise to generating myriad of degradation products including various volatile and non-volatile compounds (Nayak et al., 2015). These generated 
process-developed contaminants can deteriorate the functional, sensory attributes and nutritional value of the oils (Hua et al., 2016).

In general, there are many selections of vegetable oils available for deep-frying applications depending on their availability, traditional habits, and thermal resistance. Palm oil and soyabean oil are mostly used for domestic cooking and frying worldwide, while canola oil is commonly used in European countries and Canada (Hosseini et al., 2016). In China, palm oil is often used in fast food restaurants, i.e. Kentucky Fried Chicken (KFC), McDonald's and Dicos. This is due to palm oil's excellent thermo-oxidative stability, long shelflife, abundant phytonutrients (tocotrienols and carotenoids) and reliable supply (Kushairi et al., 2018). In 2018, Malaysia produced 19.52 million tonnes of palm oil, with annual productivity of $3.73 \mathrm{t}$ ha $^{-1} \mathrm{yr}^{-1}$ (Kushairi et al., 2019). Recently, unsaturated oils have received much attention, and among them, oil rich with monounsaturated fatty acids (MUFA) are increasingly received a broader spectrum of functionality and health benefits (Gillingham et al., 2011), with an inherently higher thermaloxidative stability compared to oils with higher polyunsaturated fatty acids (PUFA). Utilisation of high oleic oils such as high oleic rapeseed oil (canola oil), high oleic sunflower oil, high oleic peanut oil and high oleic soyabean oil continues to increase in China, especially in fast food restaurants.

Oil stability indicates the ability of oil to resist elevated temperatures during frying, as a result, the degree of saturation has become a considerable criterion for choosing suitable frying oil. A great many studies have demonstrated that oil with higher amount of saturated fatty acids and lower amount of unsaturated fatty acids exhibits better stability on frying performance irrespective of the nutritional standpoint of oil (Matthäus, 2006; Aladedunye and Przybylski, 2014). Furthermore, the number of unsaturated bonds in fatty acids plays a decisive role in the oxidation rate of oil. It is reported that the relative oxidation rate of stearic acid (18:0) to oleic acid (18:1) to linoleic acid (18:2) to linolenic acid (18:3) was 1:10:100:150 (Ahmad Tarmizi et al., 2016). Saturated fatty acid has no double bond in its fat molecule which gives the higher stability property as compared with unsaturated fatty acid. However, oil stability does not depend on the degree of saturation absolutely (Normand et al., 2006). Factors like the presence of minor components such as phytosterols, tocopherols and tocotrienols and the positioning of fatty acid in the triacylglycerols could also affect the stability of the oil (Abuzaytoun and Shahidi, 2006; Ahmad Tarmizi et al., 2016).

Therefore, the aim of the present work was to evaluate the frying performance of two commercial frying oils, which are refined, bleached and deodourised palm stearin [POs, iodine value (IV) 49] and high oleic blended oil (HOBO) consisting of a blend of high oleic sunflower oil and canola oil under following frying protocol in fast food restaurants in China. The main focus was the quality changes occurring in the two frying oils which included colour, iodine value (IV), acid value $(\mathrm{AV})$, peroxide value $(\mathrm{PV}), p$-anisidine value ( $p$-AV), total oxidation (TOTOX), carbonyl group value (CGV), fatty acids composition (FAC), total polar compounds (TPC), oxidative stability index (OSI) and tocols.

\section{MATERIALS AND METHODS}

\section{Materials}

Commercial-grade POs (IV 49) was purchased from Wilmar Co. Ltd (Shanghai, China) while HOBO consisting of a blend of high oleic sunflower oil and modified canola oil were purchased from Cargill Co. Ltd (Shanghai, China). Pre-fried French fries were provided by Inner Mongolia Lamb Weston Potato Co. Ltd (UIanqab, China). Benzene, n-hexane, n-heptane, tetrahydrofuran were chromatographic grade and purchased from Sinopharm Chemical Reagent Co. Ltd (Shanghai, China). The internal standard $\alpha-, \beta-, \gamma^{-}, \delta$-tocopherols were obtained from Sigma-Aldrich (St Louis, MO, USA) and $\alpha-, \beta-, \gamma_{-}, \delta$-tocotrienols samples were gifts from Sime Darby Plantation Company, Malaysia with purity greater than $97 \%$ by high performance liquid chromatography (HPIC). Other reagentgrade chemicals were purchased from Sinopharm Chemical Reagent Co. Ltd (Shanghai, China).

\section{Frying Protocol}

The present work was designed to simulate frying practice of fast food restaurants according to Li et al. (2019) with slight modifications, heating the oils for $12 \mathrm{hr}$ daily, with frying cycles every $12 \mathrm{~min}$ over five consecutive days. Each frying experiment was carried out using a 13-litre capacity stainless deep fryer Model OFE-28 (Yixi, Shanghai, China) with a set temperature of $170 \pm 5^{\circ} \mathrm{C}$. Each frying cycle, a batch of $200 \mathrm{~g}$ of pre-fried French fries was fried for $3 \mathrm{~min}$, and $9 \mathrm{~min}$ for the oil to remain idle before executing next frying cycle. The filtration with $60 \mathrm{~g}$ filter powder (Dallas Special Adsorbent Co., Qingdao, China) and paper (Yixi, Shanghai, China) was conducted every $6 \mathrm{hr}$ of frying. After filtering, fresh frying oil was added to replenish the used oil to the initial level in the fryer. At the end of daily frying operation, $350 \mathrm{ml}$ of each oil was collected and placed in a dark amber bottle, purged with nitrogen and kept at $-20^{\circ} \mathrm{C}$ for subsequent analyses. 


\section{Methods of Oil Analyses}

$\mathrm{AV}, \mathrm{PV}$ and TPC were determined using the AOCS Official Methods Cd 3d-63, Cd 8b-90 and Cd 20-91, respectively (AOCS, 2017). The IV and $p-A V$ of oil samples were assayed according to the ISO methods 3961:2018 and 6885:2016, respectively. The OSI of oil was measured at $110^{\circ} \mathrm{C}$ using a Metrohm 743 Rancimat (Metrohm, Switzerland) according to ISO Method 6886:2016. Determination of oil colour was carried out using a Lovibond Tintometer Model-F (Salisbury, England) following the ISO Method 27608:2010.

\section{Determination of FAC}

According to the AOCS Official Method Ce 1a-13 (2017), the FAC was analysed using gas chromatography (Agilent Technologies, Shanghai, China), coupled with a FID and polar capillary column model varian cp $7489(100.0 \mathrm{~m} \times 250$ $\mu \mathrm{m} \times 0.20 \mu \mathrm{m}$, VARIAN, Shanghai, China). The injection temperature was kept at $250^{\circ} \mathrm{C}$. The oven temperature was held for $5 \mathrm{~min}$ at $160^{\circ} \mathrm{C}$ and subsequently programmed to $210^{\circ} \mathrm{C}$ at $10^{\circ} \mathrm{C} \mathrm{min}{ }^{-1}$. Ultrahigh-purity helium was used as the carrier gas at a flow rate of $1.3 \mathrm{ml} \mathrm{min}{ }^{-1}$ and the split ratio was 50:1.

\section{Analysis of Tocol Homologues in Oil}

The concentration of tocols (tocopherols and tocotrienols) in oil was quantified following to the ISO Method 9936:2016 using a normal-phase HPLC (Agilent Technologies 1200, Shanghai, China) fitted with a fluorescence detector (Agilent Technologies, Shanghai, China) by setting excitation and emission wavelengths at $292 \mathrm{~nm}$ and at $394 \mathrm{~nm}$, respectively. A LiChrospher 100 DIOL column $(250 \mathrm{~mm} \times 4 \mathrm{~mm}$, i.d. $5 \mu \mathrm{m}$ ) was used as the stationary phase at a column temperature of $30^{\circ} \mathrm{C}$. The mobile phase used in the analysis was a mixture of 96.15:3.85 ratio of n-hexane and tetrahydrofuran at a flow rate of $1 \mathrm{ml}$ $\mathrm{min}^{-1}$. Tocopherols and tocotrienols were identified using external calibration for their individual homologue.

\section{Statistical Analyses}

All samples were analysed in triplicate, and data are presented as means \pm standard deviations (SD). Data were evaluated by single factor analyses of variance (ANOVA) using the statistical package IMB SPSS Statistics (Version 20). Statistically significant differences between means were determined by Duncan's multiple range tests for $\mathrm{p}<0.05$. Pearson correlations among the selected quality parameters were also calculated.

\section{RESULTS AND DISCUSSION}

\section{FAC}

FAC of the fresh oils used in the frying experiment are shown in Table 1. The most prominent fatty acids in fresh POs were palmitic acid $(47.92 \%)$, oleic acid $(37.49 \%)$ and linoleic acid $(9.07 \%)$, while HOBO contained much higher amount of oleic acid $(66.79 \%)$ and lesser amount of palmitic acid $(4.54 \%)$. Besides, the level of saturated fatty acids (SFA) in POs (53.44\%) was almost 10 times as high as that of HOBO (5.73), whereas only a third of the content of PUFA in HOBO was observed in POs. Oils with high amount of SFA and low amount of unsaturated fatty acids, especially PUFA, has higher oxidative stability (Ahmad Tarmizi and Ismail, 2014).

Figure 1 shows that FAC of all oils changed with frying time. Considering that linoleic acid (C18:2) is more susceptible to oxidation than palmitic acid (C16:0), the ratio of $\mathrm{C} 18: 2 / \mathrm{C} 16: 0$ is used as the indicator for oxidative deterioration in used oils (Bansal et al., 2010; Aniołowska and Kita, 2015; Li et al., 2017). As shown in Figure 1, the C18:2/C16:0 dropped progressively in $\mathrm{HOBO}$ while remained almost unchanged in POs over the frying period.

TABLE 1. FATTY ACIDS COMPOSITION (\%) OF FRESH OILS

\begin{tabular}{ccc}
\hline FAC & POs & HOBO \\
\hline C14:0 & $1.44 \pm 0.11 \mathrm{a}$ & ND \\
C16:0 & $47.92 \pm 1.19 \mathrm{a}$ & $4.54 \pm 0.15 \mathrm{~b}$ \\
C18:0 & $4.08 \pm 0.14 \mathrm{a}$ & $1.19 \pm 0.12 \mathrm{~b}$ \\
C18: 1 & $37.49 \pm 0.45 \mathrm{a}$ & $66.79 \pm 1.34 \mathrm{~b}$ \\
C18:2 & $9.07 \pm 0.04 \mathrm{a}$ & $18.70 \pm 0.08 \mathrm{~b}$ \\
C18:3 & ND & $8.78 \pm 0.09 \mathrm{~b}$ \\
SFA & $53.44 \mathrm{a}$ & $5.73 \mathrm{~b}$ \\
MUFA & $37.49 \mathrm{a}$ & $66.79 \mathrm{~b}$ \\
PUFA & $9.07 \mathrm{a}$ & $27.48 \mathrm{~b}$ \\
\hline
\end{tabular}

Note: POs - palm stearin; HOBO - high oleic blended oil; SFA - saturated fatty acids; MUFA - monounsaturated fatty acids; PUFA - polyunsaturated fatty acids; FAC - fatty acids composition; ND - not detected. Different letters are significant in the same row at $\mathrm{P}<0.05$. 


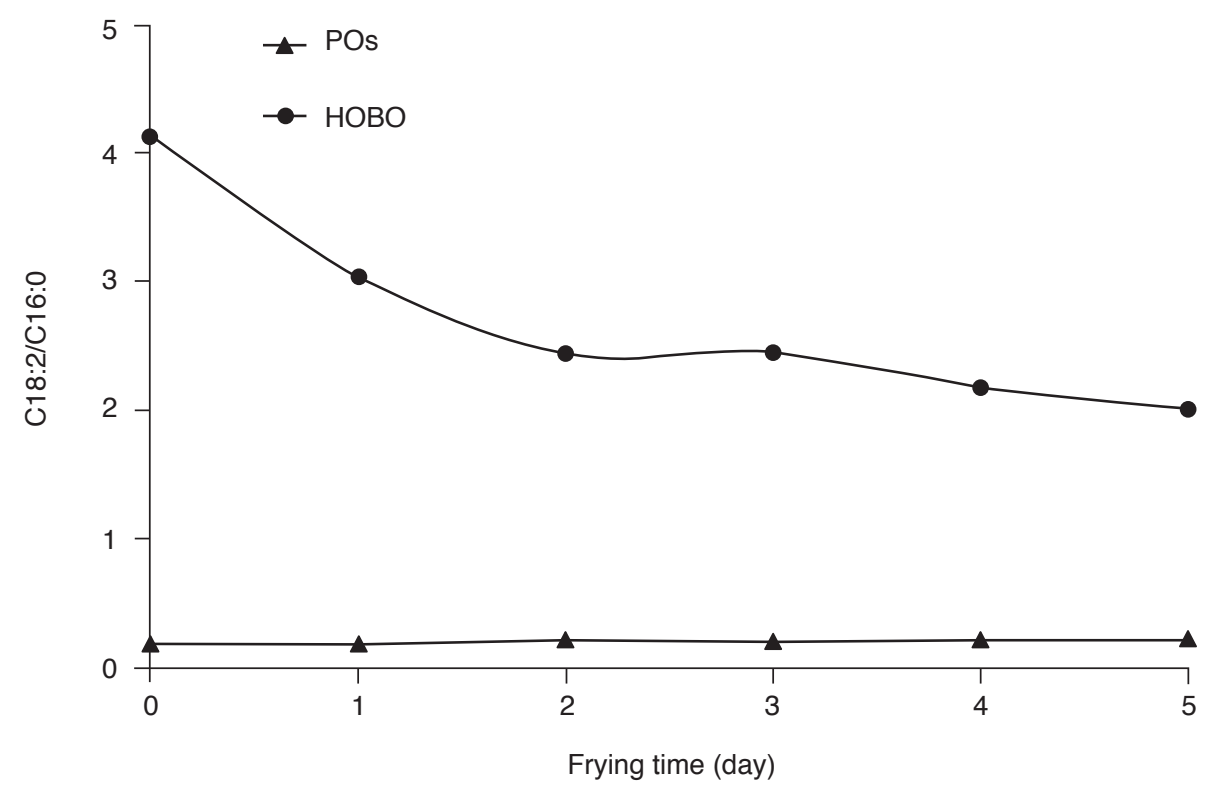

Figure 1. Changes in the ratio of C18:2/C16:0 during five days of frying in palm stearin (POs) and high oleic blended oil (HOBO).

In addition, the C18:2/C16:0 decreased by $51.46 \%$ for $\mathrm{HOBO}$, at the end of frying session. This ratio was significantly higher $(\mathrm{P}<0.05)$ than that of POs $(15.79 \%)$, which indicates that $\mathrm{HOBO}$ was susceptible towards changes in FAC when the unsaturation degree in the oil degraded due to oxidation during frying.

\section{Changes in IV}

The IV is used as the indicator to measure the degree of oil unsaturation. A decrease in IV can be due to the destruction of unsaturated bonds of oil by oxidation and polymerisation (Abdulkarim et al., 2007). Figure 2 shows the changes in IV during five consecutive days of frying. The initial IV for POs (49.42) was almost unchanged over five days of frying, while $\mathrm{HOBO}$ showed gradual decrease in the IV (from 105.63 to 95.36) during the frying period, which was in good agreement with its reduction in unsaturated fatty acids (data not shown). This meant that POs was less susceptible to oxidation than $\mathrm{HOBO}$.

\section{Colour}

Figure 3 shows the changes of oils colour across five days of frying. POs and $\mathrm{HOBO}$ darkened with frying time. Palm oil showed a similar and moderate rate of colour darkening with that of $\mathrm{HOBO}$ during the first three days before starting to accelerate for the remaining frying days (Figure 3). Darkening of oils during frying is due to the formation of nonvolatile decomposition products (Abdulkarim et al., 2007). However, colour is recognised as a prejudiced parameter used for rapid monitoring of the quality of frying oil in the food industry. As the trace pigments and tocopherols, particularly tocotrienols are prone to formation of red-brown quinone polymers which may also affect the colour of frying oils. Formation of some carotenoid oxidation products and polymerised acylglycerols may also contribute to the higher colour observed for POs (Aladedunye and Przybylski, 2014). This may be a good explanation why the initial and end of POs colour was higher despite the rate of colour darkening was comparable to that of $\mathrm{HOBO}$.

\section{Changes in AV}

AV principally measures the level of FFA in oil is usually used to indicate the degree of oil deterioration owing to hydrolysis of triglyceride and/or degradation of hydroperoxide (Matthäus, 2006). Figure 4 shows changes in $\mathrm{AV}$ for all oils during five days of frying. Increase in $\mathrm{AV}$ for both POs and HOBO was observed across frying time. However, the $\mathrm{AV}$ of both frying oils met the standards in China and Netherlands of no greater than $4.5-5.0 \mathrm{mg}$ $\mathrm{KOH} \mathrm{g}^{-1}$ even after at the end of frying session (Day 5), mainly attributed by systematic filtering and replenishing of fresh oil during frying (Aladedunye and Przybylski, 2009). At the end of frying, the AV for POs was relatively higher compared to $\mathrm{HOBO}$.

Generally, the degree of FFA through hydrolysis is too low, and poorly correlated with the quality of the food, thus, AV is not suitable to be used alone as quality indicator for frying oils (Matthäus, 2006; Aladedunye and Przybylski, 2014). However, it can be a good indicator of the extent of oil adulteration if used in combination with other methods (Abdulkarim et al., 2007). 


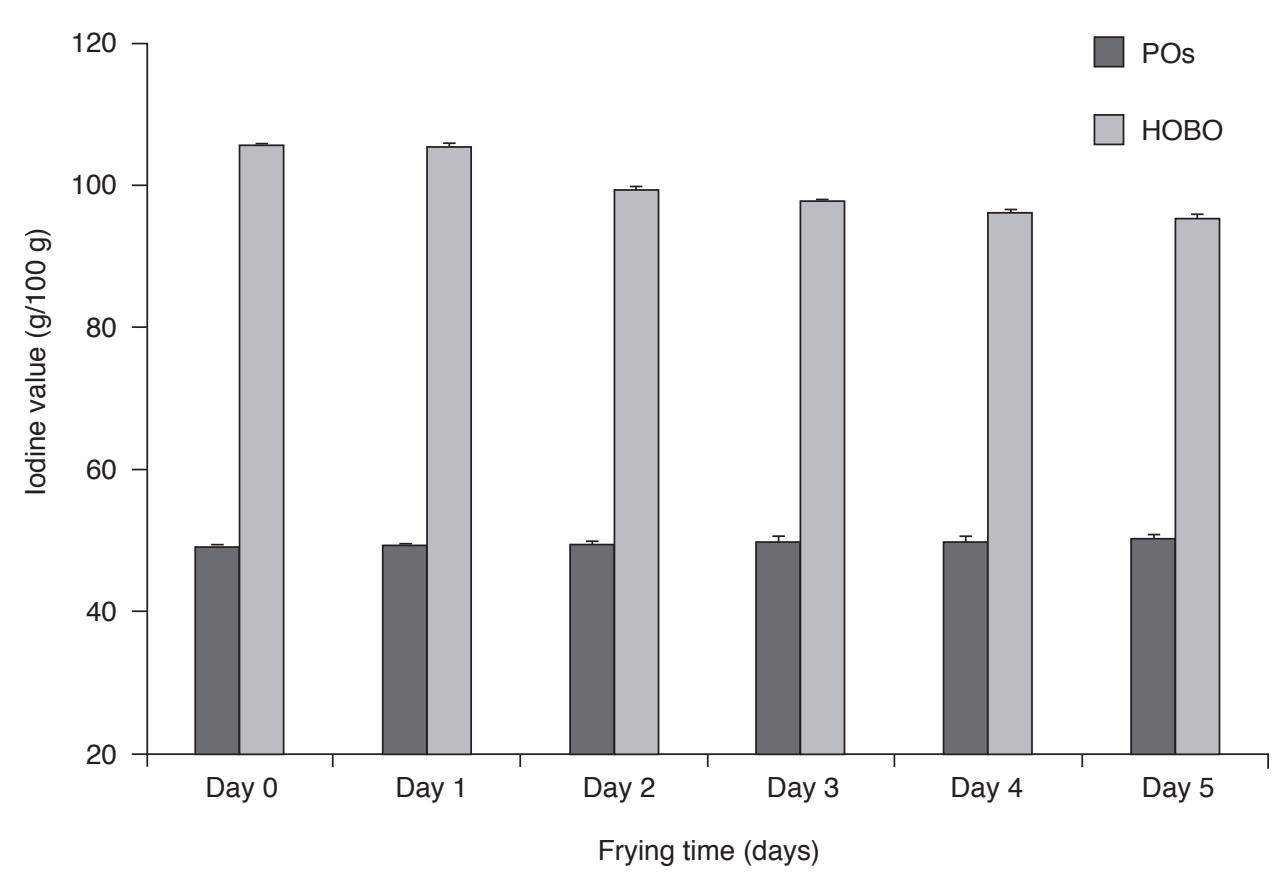

Figure 2. Changes in iodine value (IV) during five days of frying in palm stearin (POs) and high oleic blended oil (HOBO).

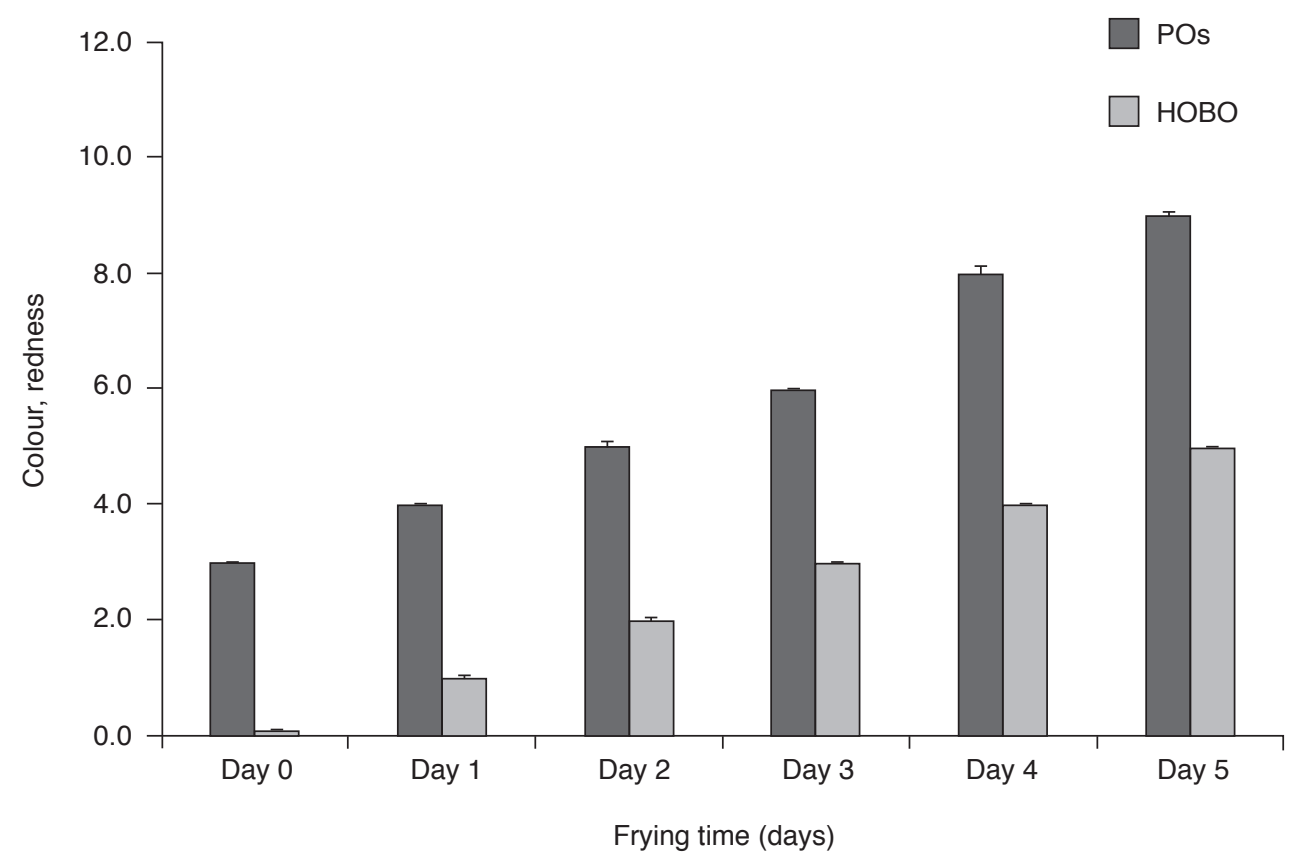

Figure 3. Changes in colour during five days of frying in palm stearin (POs) and high oleic blended oil (HOBO).

\section{Changes in PV}

Figure 5 shows the changes in PV of both oils for five days of successive frying. It was found that the PV of both oils increased drastically during the first day of frying and started to exhibit slight fluctuation thereafter. PV is considered as primary oxidation constituents of heated oil. Increase in the PV during frying period indicates the formation of hydroperoxides due to early stage of oxidation. However, the hydroperoxides are unstable and prone to decompose into carbonyl and aldehyde compounds during elevated frying temperature. At frying temperatures, hydroperoxides decompose to form carbonyl and aldehydic compounds resulting in PV increment (Abdulkarim et al., 2007). Therefore, PV appeared fluctuant throughout the frying process. However, PV alone is not a sole 


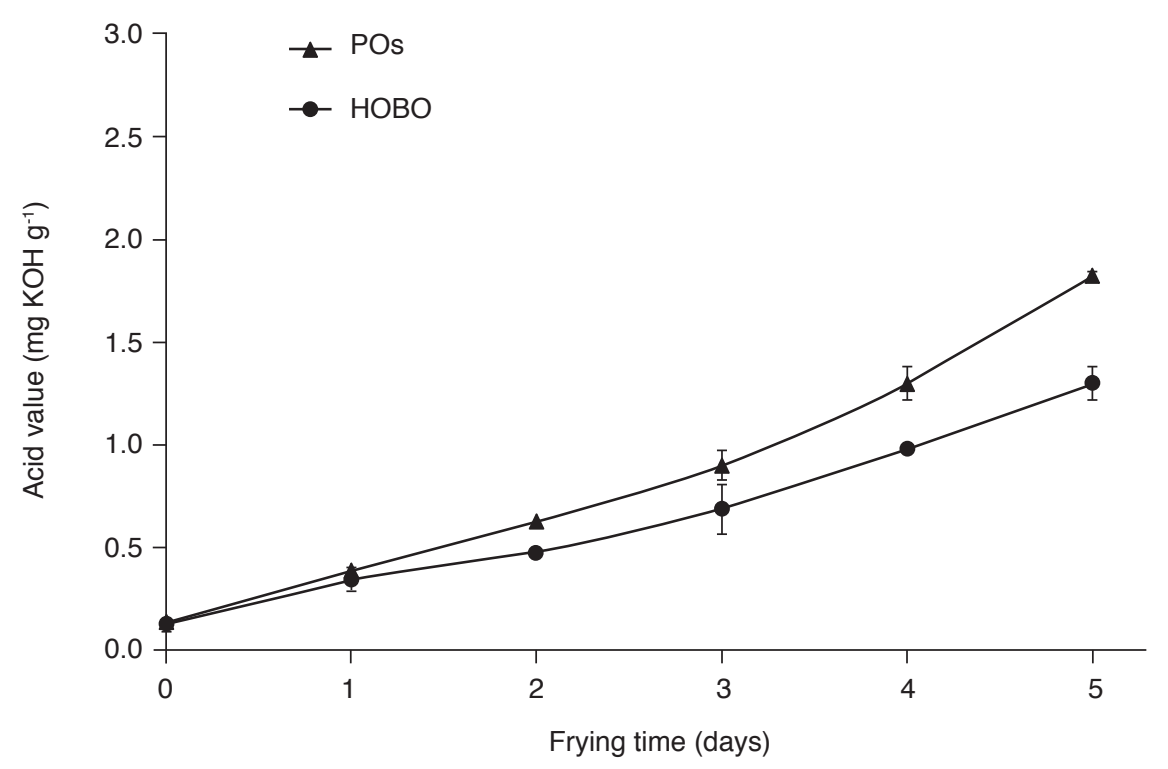

Figure 4. Changes in acid value (AV) during five days of frying in palm stearin (POs) and high oleic blended oil (HOBO).

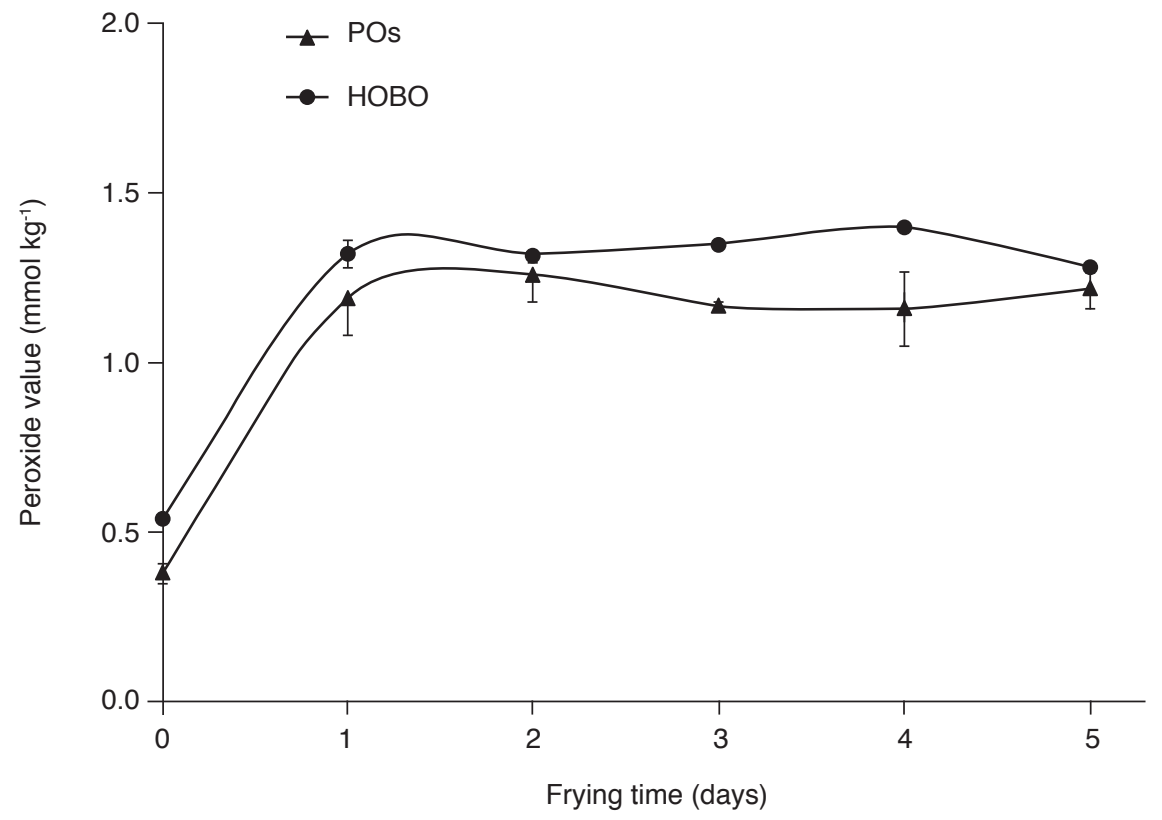

Figure 5. Changes in peroxide value (PV) during five days of frying in palm stearin (POs) and high oleic blended oil (HOBO).

parameter to assess the extent of oil deterioration. In this respect, the amount of hydroperoxides in the oil cannot be fully used to estimate oil deterioration.

\section{Changes in $p$-AV}

Thermal decomposition of hydroperoxides during frying will further generate a considerable amount of secondary oxidation components of which carbonyl compounds being the most prominent among others (Nayak et al., 2016).
Thus, $p-\mathrm{AV}$ is a good indication of the level of nonvolatile aldehydes in the frying oil (Aladedunye and Przybylski, 2014). As shown in Figure 6, this observation can be supported by the trend of $p-\mathrm{AV}$ with frying time where it remained plateaued and stayed within 24-28 unit for POs and 39-45 unit for $\mathrm{HOBO}$ after a sharp increase for the first two days of frying. These results were similar to the study done by Ahmad Tarmizi et al. (2019), where the $p$-AV of vegetable oils including palm olein, soyabean oil, canola oil and sunflower oil reached a plateau after the third day of frying. 


\section{Changes in TOTOX Value}

While PV evaluates the primary oil oxidation, $p$-AV measures the secondary oil oxidation. Therefore, $p-\mathrm{AV}$ is often used in the industry in conjunction with $\mathrm{PV}$ to calculate the total oxidation or TOTOX (2PV $+p-\mathrm{AV})$ value (Shahidi and Wanasundara, 2002). The transient of $p-\mathrm{AV}$ is shown in Figure 7. After the fifth day of frying, the increment in TOTOX value for POs (22.61 unit) was almost two-fold lower than $\mathrm{HOBO}$ (44.16 unit), which further confirmed that POs is more resistant against oxidation compared to HOBO. Higher TOTOX value in $\mathrm{HOBO}$ can be explained by their high percentage of unsaturation, especially PUFA. HOBO contains higher amounts of linoleic and linolenic acids, while POs has higher degree of saturation (palmitic acid) and MUFA, which gives the latter to pose better oxidative stability and lower TOTOX value.

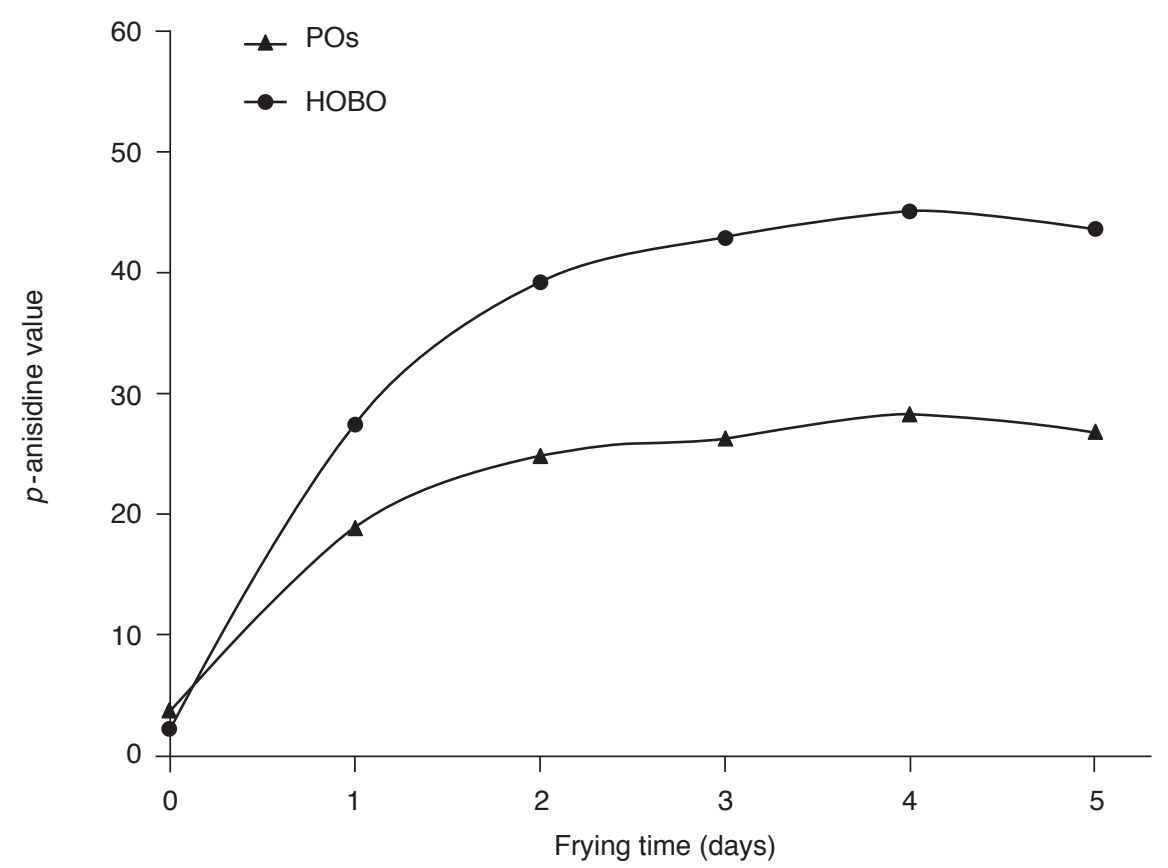

Figure 6. Changes in p-anisidine value (p-AV) during five days of frying in palm stearin (POs) and high oleic blended oil (HOBO).

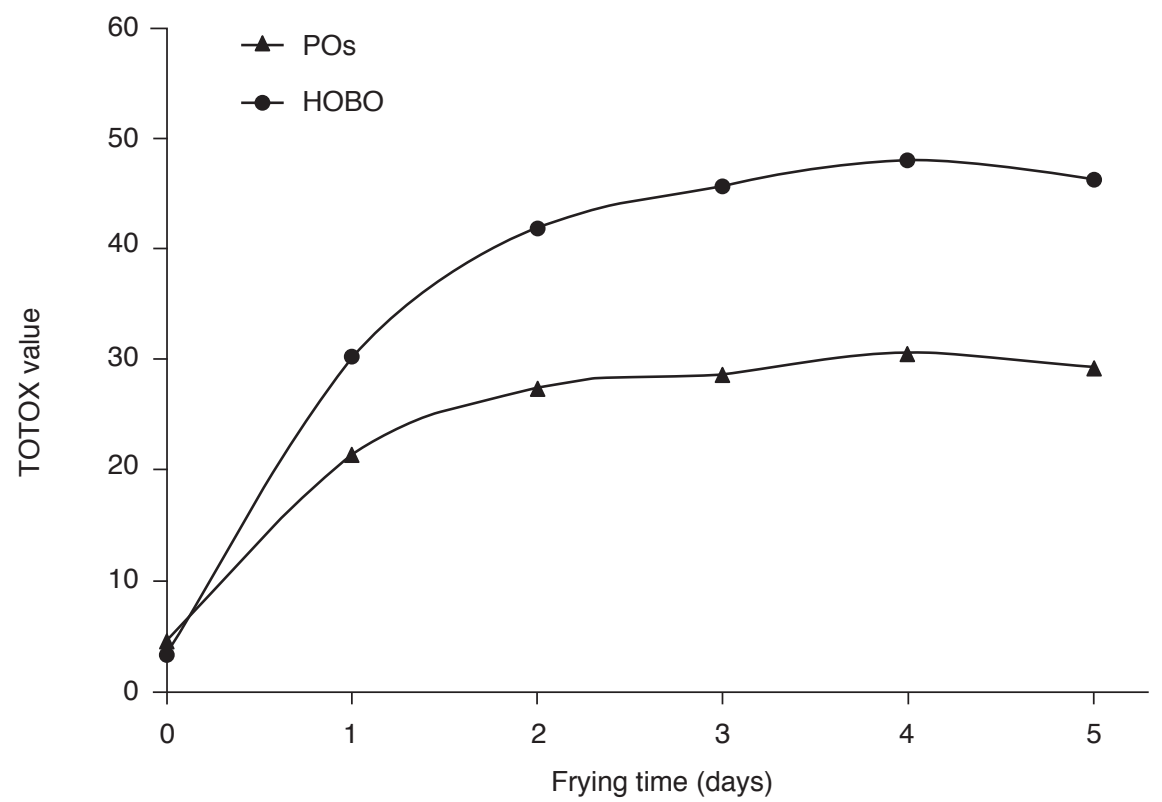

Figure 7. Changes in total oxidation (TOTOX) during five days of frying in palm stearin (POs) and high oleic blended oil (HOBO). 


\section{Changes in CGV}

CGV is useful to evaluate the quality of frying oils as it indicates the quantity of total carbonyl compounds from thermal oxidation, hydrolysis, and polymerisation during frying (Zhang et al., 2015). These compounds often result in unpleasant and rancid flavours and reduce the nutritional value of fried foods (Farhoosh and Moosavi, 2008). As shown in Figure 8, a fluctuant increase in CGV with the rising frying time was found for the two frying oils. However, the CGV of POs and HOBO still kept low level with a maximum value of $15.52 \mathrm{meq}$ $\mathrm{kg}^{-1}$ and $18.35 \mathrm{meq} \mathrm{kg}^{-1}$, respectively during frying. This levelling of CGV can be due to the addition of fresh oil every day after filtering and transfer of these carbonyl compounds into fried fries; however, their degradation should not be ignored. Moreover, the CGV in POs was significantly lower than that in $\mathrm{HOBO}$, indicating that lesser total carbonyl compounds were produced in POs.

\section{Changes in TPC}

Measurement of TPC is recognised as one of the most accurate assessments of the thermo-oxidative degradation of frying oils since the measured components are non-volatile and are representative of the major reactions occurring during frying ( $\mathrm{Li}$ et al., 2019; 2017). As shown in Figure 9, the amounts of TPC increased linearly with frying time irrespective of oil type. The level of $24 \%-27 \%$ TPC has been suggested as the maximum limit for frying oil to be edible in many European countries (Waghmare et al., 2018). Both frying oils met the requirement and exhibited good frying stability. In addition, the rate of TPC formation in POs was lower than that of HOBO despite a significant higher TPC was observed in POs during the whole frying period mainly due to its higher level of diglyceride ( $\mathrm{Li}$ et al., 2019). Ahmad Tarmizi et al. (2019) have also revealed that dependency to TPC measurement alone in arbitrating the quality and safety of oils can be misleading especially for those oils including palm oil and rice bran oil that are rich of significant level of natural diacylglycerols. Further studies on the analysis of polar compound fractions are needed to avoid deceptive perception on the state of oil deterioration by judging from the amount of TPC alone.

\section{Changes in OSI}

The OSI or induction period, an expression of the stability of oils to resist oxidation, provides dynamic measures of oil stability during storage and heating (Ahmad Tarmizi and Ismail, 2008). As shown in Figure 10, the induction period of POs decreased rapidly for the first two days of frying before remaining constant thereafter. This may be attributed to the significant level of tocotrienols in POs that accounts for almost $80 \%$ of the total tocols content. Tocotrienols with three double bonds at the side chain was more susceptible to deterioration compared to their tocopherols counterpart when exposed to excessive heat (Ahmad Tarmizi et al., 2016).

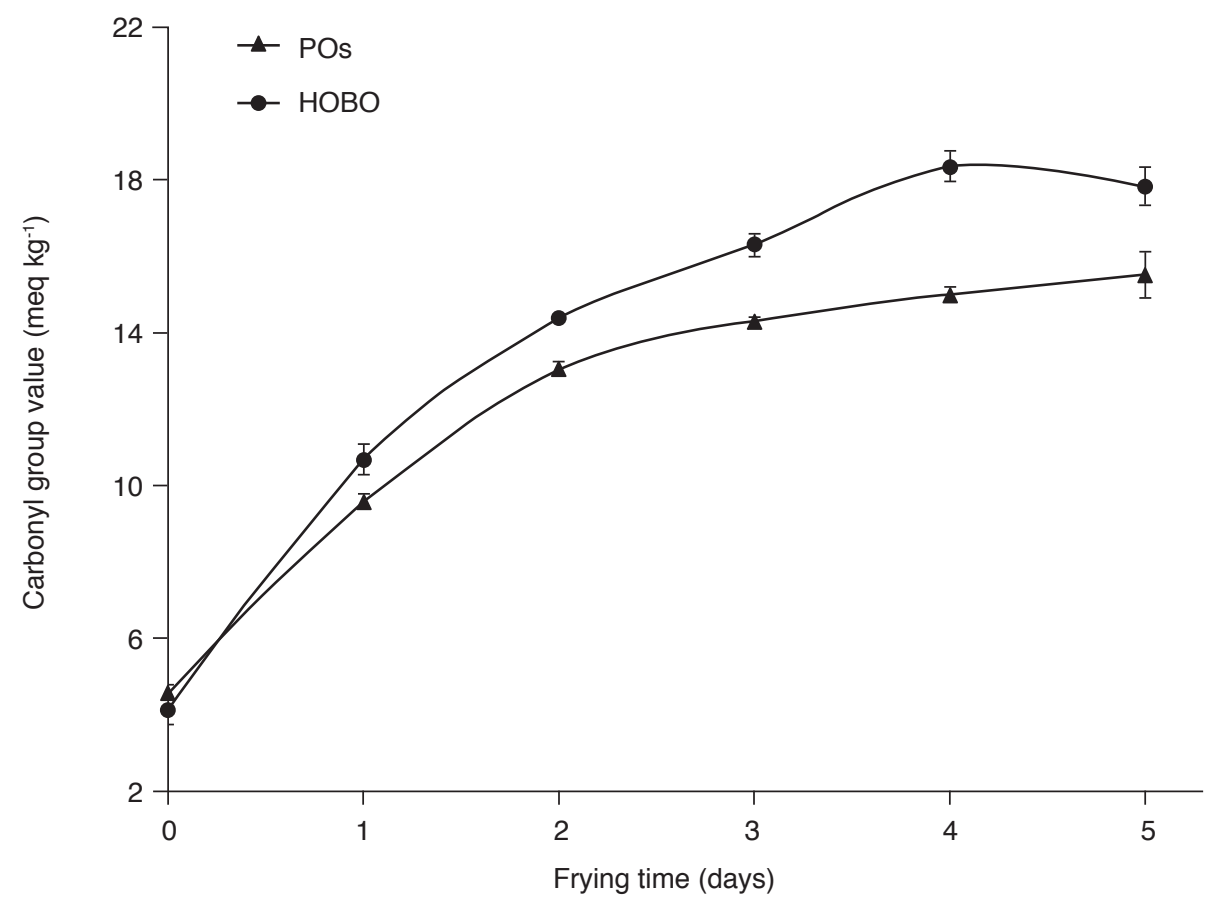

Figure 8. Changes in carbonyl group value (CGV) during five days of frying in palm stearin (POs) and high oleic blended oil (HOBO). 


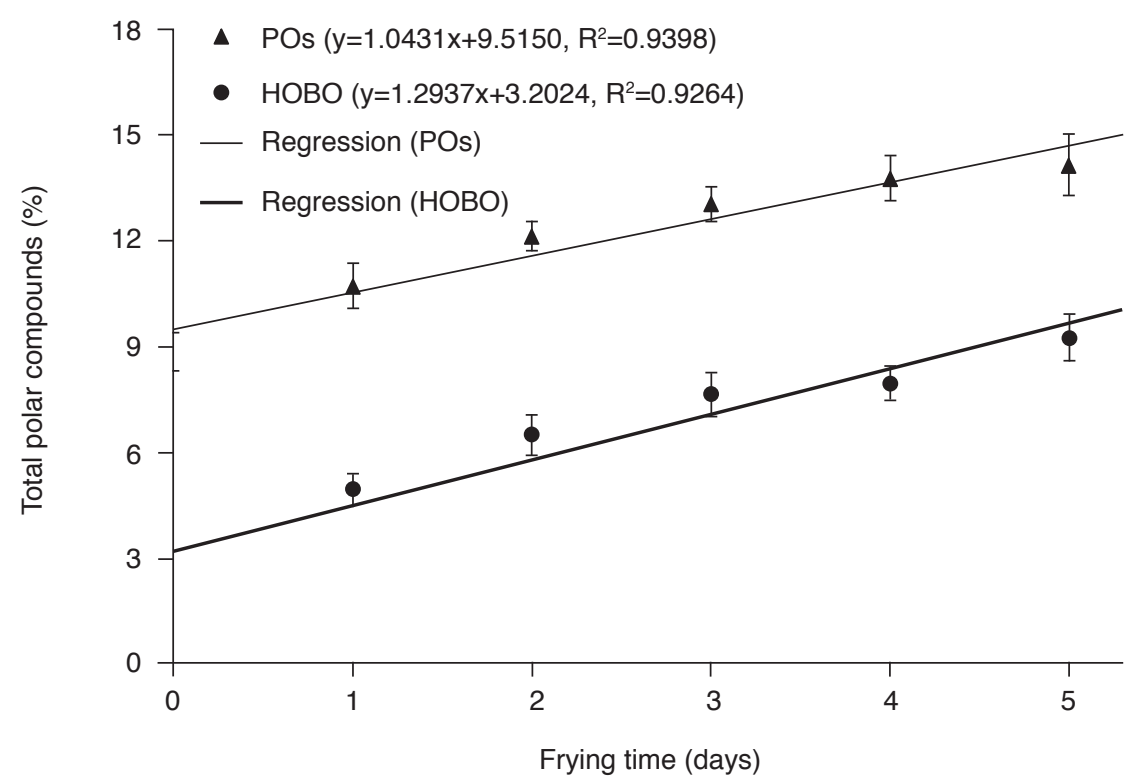

Figure 9. Changes in total polar compounds (TPC) during five days of frying in palm stearin (POs) and high oleic blended oil (HOBO).

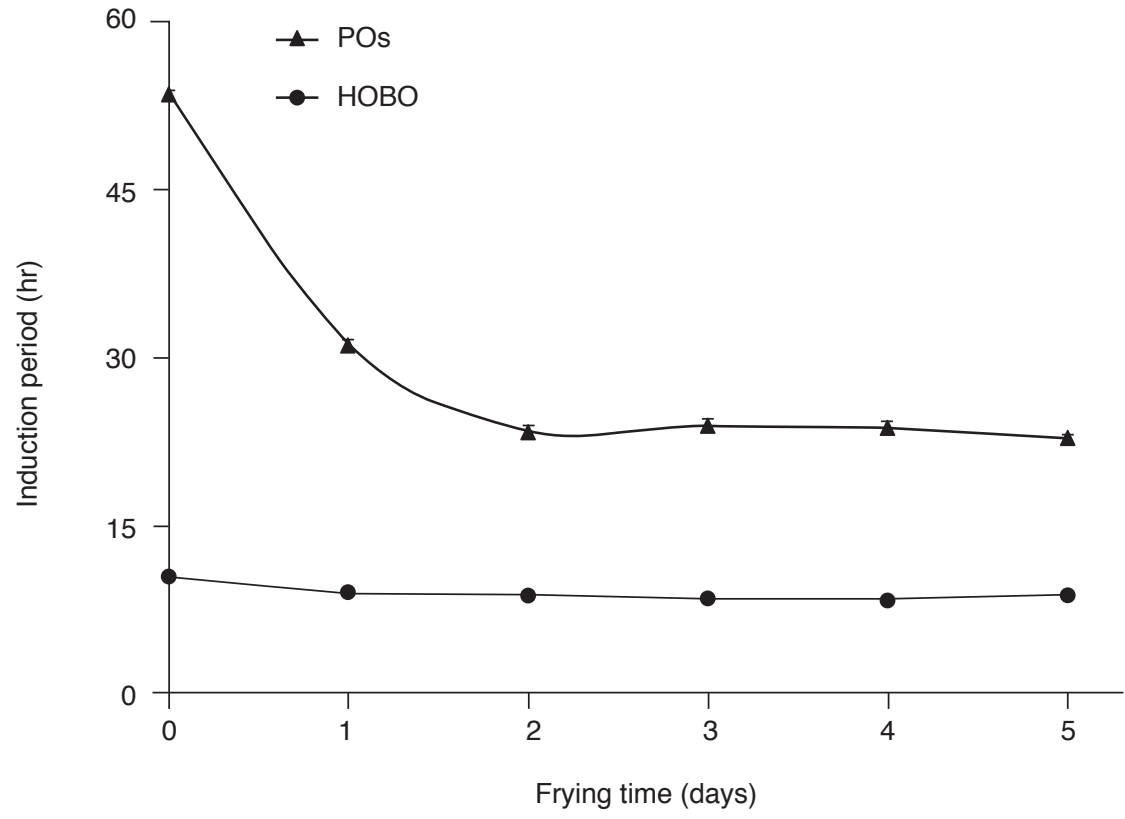

Figure 10. Changes in oxidative stability during five days of frying in palm stearin (POs) and high oleic blended oil (HOBO).

However, POs consistently displayed significantly higher induction period than $\mathrm{HOBO}$, regardless of the frying time, indicating that POs exhibited better resistance against oxidation than $\mathrm{HOBO}$. Moreover, after the fifth day of frying, the induction period of POs was still above $22 \mathrm{hr}$, which was more than three times as high as that of $\mathrm{HOBO}$ (averaging at 7 $\mathrm{hr}$ ). OSI is strongly associated to FAC before frying but highly related to the synergistic properties of antioxidants during frying (Ahmad Tarmizi and Ismail, 2014). Lower induction period in $\mathrm{HOBO}$ was due to the high percentages of unsaturated fatty acids, especially PUFA.

\section{Changes in Tocol Homologues}

Tocols which comprise of tocopherols and tocotrienols, act as antioxidants. The tocols level and isomeric constituents have been reported to influence the stability of frying oils (Warner and Moser, 2009; Romero et al., 2007). The changes in the amounts of tocopherols and tocotrienols during frying are shown in Table 2. The initial tocols content in POs was $618 \mathrm{mg} \mathrm{kg}^{-1}$, almost the same as HOBO (624 $\mathrm{mg} \mathrm{kg}^{-1}$ ), and different isomeric constituents were observed in POs and HOBO. HOBO contained only three tocopherols, while POs contained more than 
three-quarter of tocotrienols from the total tocols. Different forms of tocopherols exhibited different degrees of antioxidant effectiveness (Karmowski et al., 2015). Previous study has reported that the order of antioxidant activity of different tocotrienol isomers in vitro was $\delta>\gamma>\beta>\alpha$ (Normand et al., 2001). The tocotrienols are better antioxidants than the corresponding tocopherols, devoid of double bonds in the side chain, which is helpful to the stability of frying oil (Rossi et al., 2007).

Tocopherols and tocotrienols exhibited different stabilities for the two oils during frying. The order of degradation rate of tocol homologues after five days of simulated deep fat frying was $\gamma$-tocotrienol $(69.71 \%)>\alpha$-tocotrienol $(46.50 \%)>\delta$-tocotrienol $(38.20 \%)>\alpha$-tocopherol $(19.14 \%)>\beta$-tocotrienol $(16.79 \%)$ for POs, and $\gamma$-tocopherol $(44.27 \%)$ $>\alpha$-tocopherol $(25.02 \%)>\delta$-tocopherol $(19.57 \%)$ for $\mathrm{HOBO}$. Interestingly, it appeared that frying severely affect on $\gamma^{-}, \alpha-, \delta$-tocotrienols and $\gamma$-tocopherols but lesser impact on $\alpha$-, $\delta$-tocopherols and $\beta$-tocotrienol, which is consistent with the study conducted by Ahmad Tarmizi et al. (2008) and Aladedunye and Przybylski (2014), where tocotrienols depleted faster than tocopherols when exposed to frying temperatures. It is plausible that tocotrienols are susceptible to thermal and oxidative degradations in the presence of three double bonds in the side chain (Ahmad Tarmizi et al., 2016).

\section{Correlation between Different Parameters}

A Pearson correlation coefficients (PCC) analysis was carried out to investigate the relationships between all quality parameters in deep-fried oils (Table 3). The results showed that PV, $p-\mathrm{AV}$, TOTOX and CGV were positively correlated between one to another $(\mathrm{r}=0.859$ to 0.998$)$. TPC presented positive correlation with colour, but correlated negatively with IV, tocols and C18:2/C16:0 $(\mathrm{r}=-0.870$ to -0.932). These findings agree with the study conducted by Aladedunye and Przybylski (2009) that TPC correlated well with colour and tocopherol reduction with a correlation coefficient of 0.9302 and 0.9540 , respectively for canola oil used in seven successive days' fryings of French fries. A strong negative linear correlation between 18:2/16:0 and TPC were also observed by Bansal et al. (2010) for palm olein in repeated deep frying of French fries and chicken nuggets. In addition, it is also noted that there was an apparent correlation between IV and $\mathrm{C} 18: 2 / \mathrm{C} 16: 0(\mathrm{r}=0.960)$.

\section{CONCLUSION}

In the present study, the physical and chemical characteristics of POs and $\mathrm{HOBO}$ were determined under simulated frying practice of fast food restaurants to evaluate their thermal stabilities. In general, $\mathrm{PO}$ is more stable than $\mathrm{HOBO}$ with lower values in TOTOX, ratio of linoleic acid to palmitic acid and IV. POs also showed better oxidative stability indicated by its longer induction period and lower TPC formation rate. The increase in CGV was much higher in $\mathrm{HOBO}$ than in POs. Colour and AV increased relatively faster in POs over frying time. It is interesting to investigate the relationship

TABLE 2. CHANGES IN TOCOPHEROLS AND TOCOTRIENOLS OF TWO FRYING OILS DURING FRYING

\begin{tabular}{|c|c|c|c|c|c|c|c|c|c|}
\hline \multirow[t]{2}{*}{ Frying time } & \multicolumn{9}{|c|}{ Content/(mg kg-1) } \\
\hline & $\alpha-\mathrm{T}$ & $\beta-\mathrm{T}$ & $\gamma-\mathrm{T}$ & $\delta-T$ & $\alpha-\mathrm{T} 3$ & $\beta$-T3 & $\gamma-\mathrm{T} 3$ & ס-T3 & Tocols \\
\hline \multicolumn{10}{|l|}{ POs } \\
\hline Day 0 & $132 \pm 4 a$ & ND & ND & ND & $144 \pm 11 a$ & $49 \pm 6 a$ & $242 \pm 12 a$ & $51 \pm 4 a$ & 618 \\
\hline 1 & $123 \pm 9 a b$ & ND & ND & ND & $116 \pm 8 b$ & $47 \pm 3 a b$ & $165 \pm 11 b$ & $44 \pm 6 b$ & 494 \\
\hline 2 & $116 \pm 3 b c$ & ND & ND & ND & $98 \pm 4 c$ & $46 \pm 5 a b$ & $119 \pm 7 c$ & $38 \pm 2 b c$ & 417 \\
\hline 3 & $110 \pm 12 b c$ & ND & ND & ND & $86 \pm 8 c d$ & $44 \pm 4 \mathrm{ab}$ & $93 \pm 9 d$ & $35 \pm 4 c$ & 368 \\
\hline 4 & $107 \pm 8 c$ & ND & ND & ND & $81 \pm 2 d$ & $42 \pm 3 a b$ & $80 \pm 4 e$ & $32 \pm 3 c$ & 341 \\
\hline 5 & $106 \pm 6 c$ & ND & ND & ND & $77 \pm 9 d$ & $41 \pm 2 b$ & $74 \pm 3 e$ & $31 \pm 3 c$ & 330 \\
\hline $\begin{array}{c}\text { Degradation } \\
\text { rate }(\%)\end{array}$ & 19.14 & - & - & - & 46.50 & 16.79 & 69.71 & 38.20 & 46.67 \\
\hline \multicolumn{10}{|l|}{$\mathrm{HOBO}$} \\
\hline Day 0 & $381 \pm 9 a$ & ND & $225 \pm 7 a$ & $19 \pm 1 \mathrm{a}$ & ND & ND & ND & ND & 624 \\
\hline 1 & $346 \pm 18 b$ & ND & $196 \pm 15 b$ & $18 \pm 2 \mathrm{ab}$ & ND & ND & ND & ND & 560 \\
\hline 2 & $325 \pm 14 c$ & ND & $172 \pm 8 c$ & $17 \pm 2 \mathrm{ab}$ & ND & ND & ND & ND & 514 \\
\hline 3 & $307 \pm 8 \mathrm{~cd}$ & ND & $151 \pm 11 d$ & $16 \pm 3 a b$ & ND & ND & ND & ND & 474 \\
\hline 4 & $292 \pm 12$ de & ND & $135 \pm 9$ de & $16 \pm 1 \mathrm{ab}$ & ND & ND & ND & ND & 443 \\
\hline 5 & $285 \pm 5 e$ & ND & $125 \pm 8 e$ & $15 \pm 1 b$ & ND & ND & ND & ND & 426 \\
\hline $\begin{array}{l}\text { Degradation } \\
\text { rate }(\%)\end{array}$ & 25.02 & - & 44.27 & 19.57 & - & - & - & - & 31.78 \\
\hline
\end{tabular}

Note: ND - not detected; T - tocopherol; T3 - tocotrienol. Different letters are significant in the same column at $\mathrm{p}<0.05$. 
TABLE 3. CORRELATION COEFFICIENTS OF THE PHYSICO-CHEMICAL PARAMETERS MEASURED IN FRYING OIL SAMPLES

\begin{tabular}{|c|c|c|c|c|c|c|c|c|c|c|c|}
\hline Parameters & AV & PV & $p$-AV & TOTOX & IV & CGV & TPC & OSI & Colour & Tocols & C18:2/C16:0 \\
\hline $\mathrm{AV}$ & 1 & - & - & - & - & - & - & - & - & - & - \\
\hline PV & 0.492 & 1 & - & - & - & - & - & - & - & - & - \\
\hline$p-\mathrm{AV}$ & 0.515 & $0.877^{* *}$ & 1 & - & - & - & - & - & - & - & - \\
\hline TOTOX & 0.516 & $0.886^{* *}$ & $0.998^{* *}$ & 1 & - & - & - & - & - & - & - \\
\hline IV & -0.255 & 0.176 & 0.360 & 0.355 & 1 & - & - & - & - & - & - \\
\hline $\mathrm{CGV}$ & $0.750^{\star *}$ & $0.859^{* *}$ & $0.938^{* *}$ & $0.939^{* *}$ & 0.100 & 1 & - & - & - & - & - \\
\hline $\mathrm{TPC}$ & $0.677^{*}$ & 0.274 & 0.144 & 0.149 & $-0.848^{*}$ & $0.426^{* *}$ & 1 & - & - & - & - \\
\hline OSI & -0.185 & $-0.607^{*}$ & $-0.637^{*}$ & $-0.639^{*}$ & $-0.796^{* *}$ & -0.515 & 0.433 & 1 & - & - & - \\
\hline Colour & $0.868^{* *}$ & 0.306 & 0.241 & 0.244 & $-0.690^{*}$ & 0.541 & $0.941^{* *}$ & 0.249 & 1 & - & - \\
\hline Tocols & $-0.888^{* *}$ & $-0.576^{*}$ & -0.491 & -0.496 & 0.500 & $-0.753^{* *}$ & $-0.870^{* *}$ & $-0.050^{*}$ & $-0.932^{* *}$ & 1 & - \\
\hline C18:2/C16:0 & -0.363 & 0.016 & 0.120 & 0.116 & $0.960 * *$ & -0.116 & $-0.914^{* *}$ & -0.733 & -0.757 & 0.603 & 1 \\
\hline
\end{tabular}

Note: ${ }^{*} \mathrm{p}<0.05 ;{ }^{* *} \mathrm{p}<0.01$

$\mathrm{AV}$ - acid value.

$\mathrm{PV}$ - peroxide value.

$p$-AV - $p$-anisidine value.

TOTOX - total oxidation.

IV - iodine value.
CGV - carbonyl group value.
TPC - total polar compounds.
OSI - oxidative stability index. between different parameters in deep-fried oils. The PCC results showed that significant correlation between PV, $p-\mathrm{AV}$, TOTOX and CGV, and between TPC and colour, IV, tocols and C18:2/C16:0. Results from this study indicated that POs exhibited better thermo-oxidative stability during frying compared to HOBO. The result of this study can be used by the food processes to make their judgement in choosing the suitable oil for frying.

\section{ACKNOWLEDGEMENT}

The authors wish to thank the Director-General of MPOB for permission to publish this article. Appreciation was also extended to the Head of Analytical and Quality Development Unit, Group Leader and staff of the Palm Oil Research and Technical Service Institute of MPOB (PORTSIM), People's Republic of China for their support and technical assistance.

\section{REFERENCES}

Abdulkarim, S M; Long, K; Lai, O M; Muhammad, S K S and Ghazali, H M (2007). Frying quality and stability of high-oleic Moringa oleifera seed oil in comparison with other vegetable oils. Food Chem., 105(4): 1382-1389.

Abuzaytoun, R and Shahidi, F (2006). Oxidative stability of algal oils as affected by their minor components. J. Agric. Food Chem., 54: 8253-8260.

Ahmad Tarmizi, A H; Ahmad, K; Sahri, M M and Choo, Y M (2016). Rancimat test for measuring the oxidative stability of cooking oils upon prolonged frying: Short communication. J. Oil Palm Res. Vol. 28(4): 531-535.
Ahmad Tarmizi, A H and Ismail, R (2008). Comparison of the frying stability of standard palm olein and special quality palm olein. J. Amer. Oil Chem. Soc., 85(3): 245-251.

Ahmad Tarmizi, A H and Ismail, R (2014). Use of pilot plant scale continuous fryer to simulate industrial production of potato chips: Thermal properties of palm olein blends under continuous frying conditions. Food Sci. Nutr., 2(1): 28-38.

Ahmad Tarmizi, A H; Hishamuddin, E and Abd Razak, R A (2019). Impartial assessment of oil degradation through partitioning of polar compounds in vegetable oils under simulated frying practice of fast food restaurants. Food Control, 96: 445-455.

Ahmad Tarmizi, A H; Ismail, R and Kuntom, A (2016). Effect of frying on the palm oil quality attributes - A review. J. Oil Palm Res. Vol. 28(2): 143-153.

Aladedunye, F and Przybylski, R(2009). Degradation and nutritional quality changes of oil during frying. J. Amer. Oil Chem. Soc., 86: 149-156.

Aladedunye, F and Przybylski, R(2014). Performance of palm olein and modified rapeseed, sunflower, and soybean oils in intermittent deep-frying. Eur. J. Lipid Sci. Technol., 116(4): 144-152.

Aladedunye, F A (2015). Curbing thermo-oxidative degradation of frying oils: Current knowledge and challenge. Eur. J. Lipid Sci. Tech., 117: 1867-1881.

Aniolowska, M A and Kita, A M (2016). The effect of raw materials on thermo-oxidative stability and glycidyl ester content of palm oil during frying. J. Sci. Food Agric., 96: 2257-2264. 
Aniołowska, M and Kita, A (2015). The effect of type of oil and degree of degradation on glycidyl esters content during the frying of French fries. J. Amer. Oil Chem. Soc., 92(11): 1621-1631.

AOCS (2017). Official Methods and Recommended Practices of the American Oil Chemists' Society. Seventh edition, American Oil Chemists's Society, Champaign, IL, USA. p. 1-7.

Bansal, G; Zhou, W B; Barlow, P J; Lo, H L and Neo, F L (2010). Performance of palm olein in repeated deep frying and controlled heating processes. Food Chem., 121: 338-347.

Del Re, P V and Jorge, N (2006). Behavior of vegetable oils for frying discontinuous frozen prefried products. Science Technology Aliment, 26: 56-63.

Farhoosh, S and Moosavi, S M R (2008). Carbonyl value in monitoring of the quality of used frying oils. Anal. Chim. Acta, 617: 18-21.

Gillingham, L G; Harris-Janz, S and Jones, P J H (2011). Dietary monounsaturated fatty acids are protective against metabolic syndrome and cardiovascular disease risk factors. Lipids, 46(3): 209228.

Hosseini, H; Ghorbani, M; Meshginfar, N and Mahoonak, A S (2016). A review on frying: Procedure, fat, deterioration progress and health hazards. J. Amer. Oil Chem. Soc., 93: 445-466.

Hua, H; Zhao, X; Wu, S and Li, G (2016). Impact of refining on the levels of 4-hydroxy-trans-alkenals, parent and oxygenated polycyclic aromatic hydrocarbons in soybean and rapeseed oils. Food Control, 67: 82-89.

ISO 27608 (2010). Animal and vegetable fats and oils - Determination of Lovibond colour. 8 pp.

ISO 3961 (2018). Animal and vegetable fats and oils - Determination of iodine value. 12 pp.

ISO 6885 (2016). Animal and vegetable fats and oils - Determination of anisidine value. 7 pp.

ISO 6886 (2016). Animal and vegetable fats and oils - Determination of oxidation stability (accelerated oxidation test). $13 \mathrm{pp}$.

ISO 9936 (2016). Animal and vegetable fats and oils Determination of tocopherol and tocotrienol contents by high-performance liquid chromatography. 16 pp.

Karmowski, J; Hintze, V; Kschonsek, J; Killenberg, M and Böhm, V (2015). Antioxidant activities of tocopherols/tocotrienols and lipophilic antioxidant capacity of wheat, vegetable oils, milk and milk cream by using photochemiluminescence. Food Chem., 175: 593-600.

Kushairi, A; Soh Kheang Loh; Azman, I; Elina Hishamuddin; Meilina Ong-Abdullah; Zanal Bidin Mohd Noor Izuddin; Razmah, G; Shamala Sundram and Ghulam Kadir Ahmad Parveez (2018). Oil palm economic performance in Malaysia and R\&D progress in 2017. J. Oil Palm Res. Vol. 30(2): 163-195.

Kushairi, A; Meilina Ong-Abdullah; Balu Nambiappan; Elina Hishamuddin; Mohd Noor Izuddin Zanal Bidin; Razmah Ghazali; Vijaya Subramaniam; Shamala Sundram and Ghulam Kadir Ahmad Parveez (2019). Oil palm economic performance in Malaysia and R\&D progress in 2018. J. Oil Palm Res. Vol. 31(2): 165-194.

Li, X D; Li, J W; Wang, Y; Cao, P R and Liu, Y F (2017). Effects of frying oils' fatty acids profile on the formation of polar lipids components and their retention in French fries over deep-frying process. Food Chem., 237: 98-105.

Li, X; Wu, G C; Yang, F; Meng, L L; Huang, J H; Zhang, H; Jin, Q Z and Wang, X G (2019). Influence of fried food and oil type on the distribution of polar compounds in discarded oil during restaurant deep frying. Food Chem., 272: 12-17.

Matthäus, B (2006). Utilization of high-oleic rapeseed oil for deep-fat frying of French-fries compared to other commonly used edible oils. Eur. J. Lipid Sci. Technol., 108: 200-211.

Nayak, P K; Dash, U; Rayaguru, K and Krishnan, K $R$ (2016). Physico-chemical changes during repeated frying of cooked oil: A Review. J. Food Biochem., 40(3): 371-390.

Normand, L; Eskin, N A M and Przybylski, R (2001). Effect of tocopherols on the frying stability of regular and modified canola oils. J. Oil Chem. Soc., 78(4): 369-373.

Normand, L; Eskin, N A M and Przybylski, R (2006). Comparison of the frying stability of regular and high-oleic acid sunflower oils. J. Amer. Oil Chem. Soc., 83(4): 331-334.

Romero, N; Robert, P; Masson, L; Ortiz, J; González, K; Tapia, K and Dobaganes, C (2007). Effect of $\alpha$-tocopherol, $\alpha$-tocotrienol and Rosa mosqueta shell extract on the performance of antioxidant-stripped canola oil (Brassica sp.) at high temperature. Food Chem., 104: 383-389. 
Rossi, M; Alamprese, C and Ratti, S (2007). Tocopherols and tocotrienols as free radicalscavengers in refined vegetable oils and their stability during deep-fat frying. Food Chem., 102(3): 812-817.

Shahidi, F and Wanasundara, U N (2002). Methods for measuring oxidative rancidity in fats and oils. Food Lipids: Chemistry, Nutrition and Biotechnology. Marcel Dekker, Inc., New York, USA. p. 465-482.

Waghmare, A; Patil, S; LeBlanc, J G; Sonawane, S and Arya, S S (2018). Comparative assessment of algal oil with other vegetable oils for deep frying. Algal Res., 31: 99-106.
Warner, K and Moser, J (2009). Frying stability of purified mid-oleic sunflower oil triacylglycerols with added pure tocopherols and tocopherol mixtures. J. Amer. Oil Chem. Soc., 86: 1199-1207.

Yu, L; Li J; Ding, S; Hang, F and Fan, L (2016). Effect of guar gum with glycerol coating on theproperties and oil absorption of fried potato chips. Food Hydrocoll., 54: 211-219.

Zhang, H; Ma, J; Miao, Y; Tuchiya, T and Chen, J Y (2015). Analysis of carbonyl value of frying oil by Fourier transform infrared spectroscopy. J. Oleo Sci., 64(4): 375-380. 